\title{
Study on the properties of modified starch and its feasibility in crystal dumpling skins
}

\author{
Wang Jingwen ${ }^{1}$, Oksana Melnyk ${ }^{1}$, and Olha Ihnatieva $^{1^{*}}$ \\ ${ }^{1}$ Food Technology Department Sumy National Agrarian University, Sumy, Ukraine
}

\begin{abstract}
Four kinds of modified starches that are expected to improve the quality of crystal dumpling skins were selected, namely Potato acetate starch, Potato hydroxypropyl starch, Octenyl succinate starch sodium, Acetate cassava starch. Through the determination of the physical indicators of the above four modified starches, and then explore the feasibility of applying them to crystal dumpling skins to improve their quality. Studies have found that the light transmittance of the four modified starches is higher than that of the original crystal dumpling mixed starch, which verifies the feasibility of applying these four modified starches to the crystal dumpling skin to improve its transparency and other characteristics. At the same time, it provides a certain reference value for related research on improving the characteristics of crystal dumpling skin.
\end{abstract}

\section{Introduction}

At present, quick-frozen dumplings are developing rapidly in the quick-frozen food industry, accounting for $30 \%$ of the frozen food industry [1]. However, quick-frozen dumplings will have problems such as cracking of dumpling skins and muddy soup during processing, transportation or eating, especially for crystal dumplings made of whole starch, due to the poor water holding capacity of the epidermis, it is very easy to lose water during the production process and it is easy to freeze and crack during quick freezing at $-50^{\circ} \mathrm{C}$. Therefore, it is very important to find ways to improve its quality characteristics. Modified starch is a starch derivative obtained by processing physical and chemical methods on the basis of the original starch [2]. At present, a large number of studies have shown that the application of modified starch in dumplings can improve its transparency, reduce the rate of freeze cracking and cooking loss, etc.

In view of the fact that there are more researches on the application of modified starch in quick-frozen dumplings made of flour [3-6], and there are relatively few researches on modified starch in quick-frozen crystal dumplings made of whole starch. The main research contents of this topic are: taking the commercially available crystal dumpling powder as the blank group and combining previous studies, four modified starches that are expected to improve the quality of crystal dumpling skins were selected: potato acetate starch, potato hydroxypropyl starch, octenyl succinate starch sodium, acetate cassava starch, by studying the physical properties of these starches, and then exploring the feasibility of applying it to crystal dumpling skins to improve its quality.

\footnotetext{
*Corresponding author: ignatieva10@gmail.com
}

\section{Experimental}

Wheat starch and corn starch Shandong Fanpu Food Co., Ltd. Potato acetate atarch Bolian Food Biotechnology Co., Ltd.; Potato hydroxypropyl starch Jinzun Food Ingredients Mall; Octenyl succinate starch sodium Shengda Food Business Department; Acetate cassava starch Henan Wanbang Industrial Co., Ltd:

RVA-StarchMaster2 Rapid viscosity analyzer Shanghai Ruifen Company; L-550 Desktop low-speed largecapacity centrifuge Hunan Xiangyi Company; PH-070A Electric heating constant temperature blast drying oven Shanghai Yiheng Company; Electronic balance (0.0001g) Shanghai Qingke Tianmei Company; 722 Visible spectrophotometer Shanghai Youke Company; XW-80A vortex mixer Haimen Qilin Bell Company; HH-S2 digital display constant temperature water bath Jiangsu Jinyi Company.

Determination of swelling degree and solubility of modified starch. Weigh $0.5 \mathrm{~g}$ (dry basis, denoted as $\mathrm{w}_{0}$ ) of modified starch in a dry and clean centrifuge tube, weigh $\mathrm{w}_{1}$, add $20 \mathrm{~mL}$ of distilled water, mix with a vortex mixer for $30 \mathrm{~s}$, respectively at $45,55,65,75,85,95^{\circ} \mathrm{C}$ keep in water bath for $30 \mathrm{~min}$, cool to room temperature, centrifuge at $5000 \mathrm{r} / \mathrm{min}$ for $15 \mathrm{~min}$, pour off the supernatant, weigh $\mathrm{w}_{2}$, calculate the swelling power and solubility of modified starch according to formula (1) and (2) respectively [7]. Take $5 \mathrm{~mL}$ of the supernatant and place it in a drying dish at $105^{\circ} \mathrm{C}$ and dry it to a constant weight to obtain the quality $\mathrm{w}_{3}$ of the water-soluble starch.

$$
\begin{aligned}
& \text { Swelling Power }=\frac{W_{2}-W_{1}}{\text { Dry weight of raw starch }} * 100 \% \\
& \text { So lub ility }=\frac{4 W^{3}}{\text { Dry weight of raw starch }} * 100 \%
\end{aligned}
$$

Determination of transparency of modified starch paste. Add 1\% (dry basis) modified starch suspension into 
a $20 \mathrm{~mL}$ test tube, and then place it in a $95^{\circ} \mathrm{C}$ water bath to heat for $30 \mathrm{~min}$, mix with a vortex mixer every $5 \mathrm{~min}$ for $30 \mathrm{~s}$, wait to cool to room temperature, and measure the starch paste at a wavelength of $650 \mathrm{~nm}$ light transmittance, distilled water is used as a blank [8], the light transmittance reflects the transparency of starch paste.

Determination of gelatinization characteristics of modified starch. The gelatinization characteristic value of starch is determined according to the method standard specified by the American Association of Cereal Chemists (AACC). Accurately weigh a certain amount of starch, the starch mass fraction is $6.0 \%(\mathrm{w} / \mathrm{w}, \mathrm{db})$, add it to an aluminum box containing $25 \mathrm{~g}$ of distilled water, stir evenly with a rotating paddle, and place it in an RVA-rapid viscosity analyzer for measurement.

Data processing and analysis. The statistical analysis of the data obtained in the experiment uses SPSS 24 software for statistical analysis of the data, and Origin Pro 9.1 software for drawing processing. All data are given in Mean $\pm \mathrm{SD}$, and the ANOVA method is used for the significance test. $p<0.05$ means statistically significant difference.

\section{Results and discussion}

\subsection{Modified starch swelling power and solubility}

Swelling power reflects the water absorption capacity of starch during cooking, and reflects the characteristics of amylose [9]. Amylose inhibits the expansion of starch [10]; the influence of temperature on the swelling power of mixed starch and modified starch is shown in Figure 1. Within $45^{\circ} \mathrm{C} \sim 95^{\circ} \mathrm{C}$, the overall trend is that the swelling power of modified starch increases with increasing temperature. This is because as the temperature increases, more water molecules enter the amorphous area of the starch granule. When the temperature reaches its gelatinization temperature, the swollen amorphous area accelerates the destruction of the starch crystallization area, which in turn leads to an increase in starch swelling [11-13].

Solubility reflects the magnitude of the interaction force between starch and water, and has a greater impact on the processing characteristics of starch. The dissolution of starch is mainly due to the escape of amylose from the swollen granules [14]. The solubility of the original mixed starch and the modified starch is shown in Figure 2. The solubility of the modified starch increases with the increase of temperature, and the solubility increases rapidly from $65^{\circ} \mathrm{C}$. This is because the starch is heated to absorb water and gelatinize rapidly, and high-energy water and heat destroys the internal hydrogen bonds of starch molecules, and part of the amylose gradually dissolves in water when heated, so the solubility of starch increases significantly [15-17].

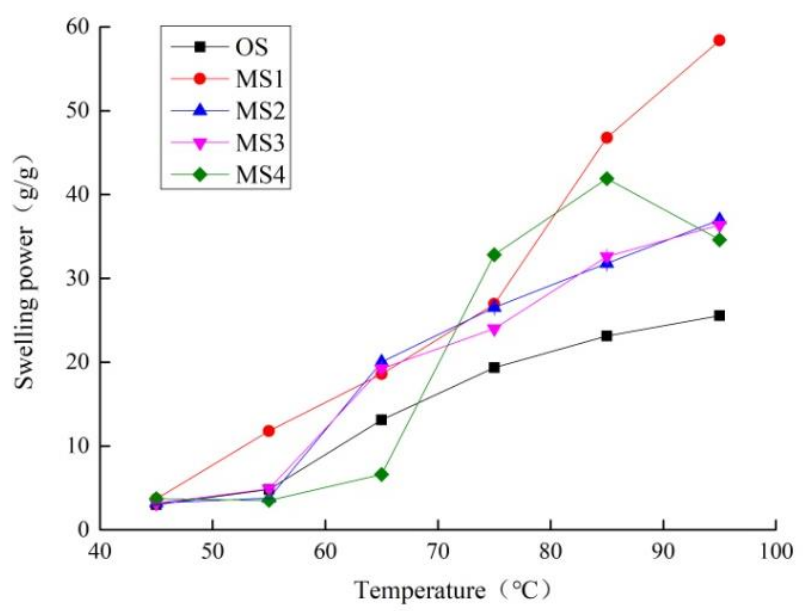

Fig. 1 The effect of temperature on the swelling power of native starch and modified starch

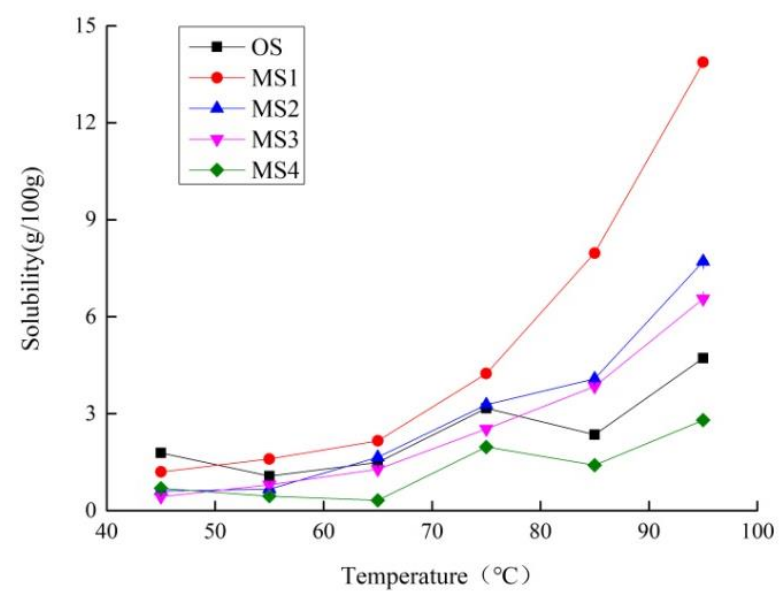

Fig. 2. The effect of temperature on the solubility of native starch and modified starch

\subsection{Transparency of modified starch paste}

The transparency of modified starch paste is reflected by light transmittance, which reflects the mutual solubility of starch and water [18-19]. The light transmittance of the original mixed starch and modified starch is shown in above Figure 3. The light transmittance of the four modified starches is higher than that of the original mixed starch OS, and the light transmittance of MS2 is the highest, followed by the light transmittance of MS3 and MS1, MS4 has the lowest light transmittance but still higher than OS. This proves that the idea of applying it to crystal dumpling skins to improve its transparency is feasible. 


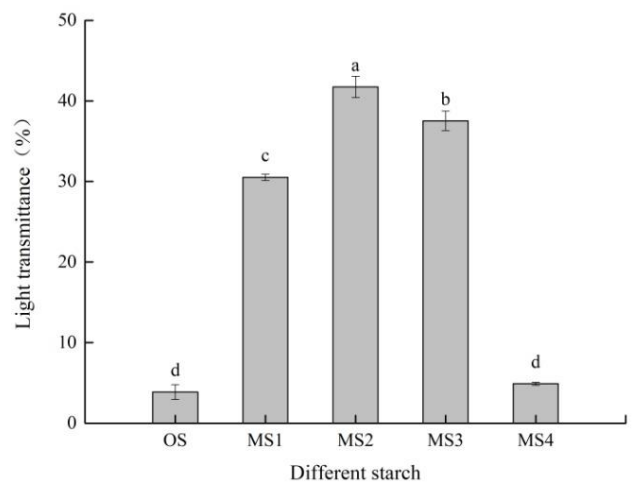

Fig. 3. Light transmittance of different native starches and modified starches(OS-wheat starch: corn starch $=3: 1$ mixed starch; MS1-potato acetate starch; MS2-potato hydroxypropyl starch; MS3- octenyl succinate starch sodium; MS4-cassava acetate starch)

\subsection{Gelatinization properties of modified} starch

The gelatinization properties of starch are an important indicator reflecting the quality of starch, which affects the appearance, texture and mouthfeel of noodle products. The gelatinization characteristics of these starches were measured by RVA. RVA measures the process from the beginning of water absorption and swelling of starch granules to the destruction of the granular structure under the action of shear force and the leaching of starch molecules [20]. The gelatinization characteristic values of different raw starches are shown

in Table 1. For the gelatinization temperature: the gelatinization temperature of the mixed powder with modified starch is higher than that of the blank group, and the gelatinization temperature except for the MS4-15\% powder which are increase with the addition of modified starch; for peak viscosity and pow viscosity: the peak viscosity and low viscosity of the mixed powder with modified starch are lower than the blank group, and increase with the increase of the content of modified starch; Final viscosity: The final viscosity of the mixed powder with modified starch added is lower than that of the blank group. For attenuation value and retrogradation value: the attenuation value and retrogradation value of the mixed powder with modified starch added are lower than the blank group, and except for the fourth modified starch, the attenuation value and retrogradation value of other blended powders decreased with the increase of modified starch content. This may be due to the preferential water swelling of modified starch, which limits the water available for wheat starch, hinders the expansion of wheat starch, and leads to the decline of the gelatinization parameters (such as peak viscosity, attenuation value and retrogradation value, etc.) of the blended starch [21].

\section{Conclusion}

Based on previous studies, it is determined that the four modified starches selected are: Potato acetate starch,

Table 1.. Effects of types and addition amount of modified starch on gelatinization characteristics of original mixed powder

\begin{tabular}{|c|c|c|c|c|c|c|}
\hline Sample & $\begin{array}{l}\text { Gelatinization } \\
\text { temperature/cp }\end{array}$ & $\begin{array}{c}\text { Peak } \\
\text { viscosity/cp }\end{array}$ & $\begin{array}{c}\text { Low } \\
\text { viscosity/cp }\end{array}$ & $\begin{array}{c}\text { Final } \\
\text { viscosity/cp }\end{array}$ & $\begin{array}{l}\text { Attenuation } \\
\text { value/cp }\end{array}$ & $\begin{array}{c}\text { Regeneration } \\
\text { value/cp }\end{array}$ \\
\hline 0 & $59.2 \pm 0.1$ & $2248.3 \pm 0.6$ & $1746.3 \pm 0.2$ & $3179.0 \pm 0.9$ & $562.0 \pm 0.1$ & $1446.0 \pm 0.4$ \\
\hline MS1-5\% & $77.7 \pm 0.3$ & $1447.3 \pm 0.0$ & $1054.0 \pm 0.7$ & $2149.7 \pm 0.0$ & $393.3 \pm 0.9$ & $1095.7 \pm 0.4$ \\
\hline MS1-10\% & $72.1 \pm 0.2$ & $1478.3 \pm 0.5$ & $1140.3 \pm 0.8$ & $2141.3 \pm 0.3$ & $338.0 \pm 0.5$ & $1001.0 \pm 0.1$ \\
\hline MS1-15\% & $71.2 \pm 0.7$ & $1493.3 \pm 0.0$ & $1157.3 \pm 0.1$ & $2142.0 \pm 0.5$ & $336.0 \pm 0.9$ & $984.7 \pm 0.5$ \\
\hline 0 & $59.2 \pm 0.1$ & $2248.3 \pm 0.6$ & $1746.3 \pm 0.2$ & $3179.0 \pm 0.9$ & $562.0 \pm 0.1$ & $1446.0 \pm 0.4$ \\
\hline MS2-5\% & $86.0 \pm 0.7$ & $1364.0 \pm 0.8$ & $971.0 \pm 0.8$ & $1996.0 \pm 0.7$ & $393.0 \pm 0.7$ & $1025.0 \pm 1.0$ \\
\hline MS2-10\% & $79.8 \pm 0.1$ & $1381.0 \pm 0.8$ & $1033.7 \pm 0.4$ & $1955.3 \pm 0.5$ & $347.3 \pm 0.0$ & $921.7 \pm 0.5$ \\
\hline MS2-15\% & $73.5 \pm 0.3$ & $1457.7 \pm 0.4$ & $1125.3 \pm 0.1$ & $1999.3 \pm 0.1$ & $332.3 \pm 0.2$ & $874.0 \pm 0.6$ \\
\hline 0 & $59.2 \pm 0.1$ & $2248.3 \pm 0.6$ & $1746.3 \pm 0.2$ & $3179.0 \pm 0.9$ & $562.0 \pm 0.1$ & $1446.0 \pm 0.4$ \\
\hline MS3-5\% & $85.6 \pm 0.5$ & $1378.7 \pm 0.5$ & $980.0 \pm 1.0$ & $2031.7 \pm 0.3$ & $398.7 \pm 0.5$ & $1051.7 \pm 0.5$ \\
\hline MS3-10\% & $75.8 \pm 0.2$ & $1452.0 \pm 0.5$ & $1068.7 \pm 0.9$ & $2045.7 \pm 0.1$ & $383.3 \pm 0.5$ & $977.0 \pm 0.6$ \\
\hline MS3-15\% & $74.6 \pm 0.0$ & $1510.0 \pm 0.5$ & $1165.3 \pm 0.5$ & $2066.0 \pm 0.3$ & $344.7 \pm 0.4$ & $900.7 \pm 0.3$ \\
\hline 0 & $59.2 \pm 0.1$ & $2248.3 \pm 0.6$ & $1746.3 \pm 0.2$ & $3179.0 \pm 0.9$ & $562.0 \pm 0.1$ & $1446.0 \pm 0.4$ \\
\hline MS4-5\% & $86.9 \pm 0.5$ & $1265.3 \pm 0.3$ & $897.3 \pm 0.2$ & $2000.0 \pm 0.8$ & $368.0 \pm 0.1$ & $1102.7 \pm 0.7$ \\
\hline MS4-10\% & $87.0 \pm 0.7$ & $1304.0 \pm 0.2$ & $922.0 \pm 1.0$ & $2077.3 \pm 0.6$ & $382.0 \pm 0.0$ & $1155.3 \pm 0.0$ \\
\hline MS4-15\% & $87.4 \pm 0.8$ & $1365.3 \pm 1.0$ & $929.7 \pm 0.5$ & $2118.0 \pm 1.0$ & $435.7 \pm 0.5$ & $1188.3 \pm 0.1$ \\
\hline
\end{tabular}


Potato hydroxypropyl starch, Octenyl succinate starch sodium, Acetate cassava starch. The properties are compared and analyzed by measuring their physical indicators. The difference between them is found: Compared with the original mixed powder, the light transmittance of the four modified starches is higher than that of the original mixed starch. Among them, potato hydroxypropyl starch has the highest transparency; among the four modified starches, acetate cassava starch has the lowest light transmittance but is still higher than the transparency of the original mixed starch. The research results of gelatinization characteristics show that: modified starch has a great influence on the gelatinization characteristics of the original mixture: the gelatinization temperature is increased, and the remaining gelatinization parameters (peak viscosity, low viscosity, final viscosity, attenuation value, and retrogradation value) are reduced. This verifies the feasibility of the idea of applying these four modified starches to crystal dumpling skins, and at the same time provides a certain reference for related research on improving the characteristics of crystal dumpling skins.

\section{References}

1. Z. Wenye. "Frozen convenience food processing technology and inspection, Beijing: Chemical Industry Press (2005)

2. Z. Yanping, Modified starch production and application [M]. Beijing: Chemical Industry Press (2007)

3. L. Wenjuan, H.. Shaokai, C. Yu et al. China Food Additives, 12, 60-64 (2015)

4. Y. Tuo, H. Juan, L. Yajie, etc. Gourmet Research, 36(02), 23-27 (2019)

5. J. Colive, R.A. Carvalho. Industrial Crops \& Products, 95 (2017)
6. Z. Sen, Z. Chunhua, H. Weixia, etc. Food Research and Development, 37(23), 21-25 (2016)

7. O.S. Lawal, K.O. Adebowale, B.M. Ogunsanwo, et al. International Journal of Biological Macromolecules, 35, 71-79 (2005)

8. F.O Onofre., Y.J Wang. International Journal of Pharmaceutics, 385(1-2), 104-112 (2010)

9. S.V Gomand., L. Lamberts, R.G.F Food Hydrocolloids, 24, 424-433 (2010)

10. R.F.Tester, W.R Morrison,. Cereal Chemistry, 67(6), 551-557 (1990)

11. Q.S. Lawal, J.Food Hydrocolloids, 23, 415-425 (2009)

12. S.G Choi, Starch/Stärke, 56, 181-189 (2004)

13. H.-J.Chung, , K-S Woo, S.-T Lim, Carbohydrate Polymers, 55, 9- 15 (2004)

14. J.S Lee., R.N. Kumar, H.D. Rozman,et al. Food Chemistry, 91, 203-211 (2005)

15. M. Xiangyan, Research on the physical and chemical properties of yellow rice starch. Chongqing: Southwest University (2008)

16. B. Berton, J. Scher, F. Villieras, et al. Power Technology, 128(2-3), 326-331 (2002)

17. R.S. Policegoudra, S.M Aradhya. Food Hydrocolloids, 22(4), 513-519 (2008)

18. D. Shuangkui, Z. Liqing, Y. Xiuzhu et al. Journal of the Chinese Cereals and Oils Association, 26(3), 3439 (2010)

19. D. Xianfeng, X. Shiying, W. Zhang. Transactions of the Chinese Society of Agricultural Engineering, 18(1), 129-132 (2002)

20. D. Cozzolino, S. Roumeliotis, J. Eglinton, Food Research International, 51(2), 444-449 (2013)

21. L. Ying, X. Zhiping. Agricultural Machinery, 5, 91-95 (2011) 